\title{
Las complicaciones de la herida quirúrgica en el trasplante renal
}

\author{
$M^{\alpha}$ Teresa Zudaire Arana* \\ $M^{\alpha}$ Victoria Cabeza Guerra* \\ Raquel Menezo Viadero** \\ Natalia Arias Ramos* \\ Rosa Alonso Nates***
}

\author{
*Enfermera hospitalización \\ **Enfermera hemodiálisis \\ ***Supervisora \\ Servicio de Nefrología. Hospital Universitario \\ Marqués de Valdecilla. Santander
}

\section{RESUMEN}

Observamos un empeoramiento en la evolución de las heridas quirúrgicas en el postoperatorio del trasplante renal. El comienzo de este problema se presenta al inicio del tratamiento con rapamicina combinado con otros inmunosupresores que ya utilizábamos.

Objetivo. Valorar si se corresponde con la observación de enfermería, que el empeoramiento en la evolución de la herida quirúrgica del postrasplante renal, está en relación con el uso de rapamicina como inmunosupresor, añadido a los habituales factores de riesgo.

Material y método. Estudio epidemiológico observacional de cohortes retrospectivo. Se estudió a un total de 56 pacientes trasplantados en nuestra unidad, desde el año 1999 hasta el 2003. Se seleccionan 30 pacientes con la pauta habitual de inmunosupresión: corticoides, ciclosporina o tacrolimus, micofenolato mofetil, etc. y a 26 pacientes con similar tratamiento, pero asociado a la rapamicina.

Resultados. La relación entre los días de exudado de la herida quirúrgica, en el postoperatorio de los pacientes del grupo tratado con rapamicina, es estadísticamente significativa en relación con el grupo que no lo toma, con una $p=0.016(p<0,05)$ en $U$ de Mann Whitney. No siendo significativas las diferencias entre otras variables estudiadas.

PALABRAS CLAVE: TRASPLANTE RENAL INMUNOSUPRESORES

RAPAMICINA

SIROLIMUS

CICATRIZACIÓN

EXUDADO

HERIDA

\section{COMPLICATIONS OF THE SURGICAL WOUND IN KIDNEY TRANSPLANTS}

\section{SUMMARY}

We note a worsening in the evolution of the surgical wounds in the post-operative kidney transplant. The problem starts at the beginning of treatment with rapamicin combined with other immunosuppressors we already used.

Objective. To evaluate whether there is correspondence with the nursing observation that the poorer evolution of the postoperative surgical wound of kidney transplants are related to the use of rapamicin as an immunosuppressor, added to the habitual risk factors.

Material and method. Retrospective observational epidemiological study of cohorts. A total 56 patients transplanted in our unit were studied from 1999 to 2003. Thirty patients were selected with the usual immunosuppression tendency: corticoids, cyclosporin 
or tacrolimus, mofethyl micophenolate, etc. and 26 patients with a similar treatment, but associated with rapamicin.

Results. The relationship between the days of exudation of the surgical wound in the postoperative period of the patients of the group treated with rapamicin is statistically significant in relation to the group that did not take it, with $p=0.016(p<0.05)$ in $U$ by Mann Whitney. The difference between other variables studied was not significant.

$\begin{array}{ll}\text { KEYWORDS: } & \text { KIDNEY TRANSPLANT } \\ & \text { IMMUNOSUPRESSORS } \\ & \text { RAPAMICIN } \\ & \text { SIROLIMUS } \\ & \text { CICATRISATION } \\ & \text { EXUDATE } \\ & \text { WOUND }\end{array}$

\section{INTRODUCCIÓN}

Una de las alternativas al tratamiento de la enfermedad renal es el trasplante, terapéutica no exenta de riesgos, pero sin ninguna duda, de elección en los pacientes renales. Han sido numerosos los cambios que la ciencia ha introducido en el tratamiento y seguimiento de los trasplantes, en estos años de evolución. El tratamiento inmunosupresor ha sido uno de ellos, se ha hecho mucho más agresivo y selectivo y, por otro lado, tanto los donantes como los receptores han experimentado cambios importantes, con más factores de riesgo añadidos.

Recientemente, la enfermería del servicio de nefrología ha observado un empeoramiento de las heridas quirúrgicas, en el post-operatorio inmediato del trasplante renal. Nuestro equipo tiene una amplia experiencia en el seguimiento de los pacientes trasplantados y esta situación nos recordaba décadas anteriores en las que la herida quirúrgica tardaba en cicatrizar y esto se producía por el empleo de altas dosis de esteroides.

Comenzamos a analizar este problema al darnos cuenta que no sucedía en todos los pacientes, y parecía corresponder al inicio de la utilización de la rapamicina (sirolimus), asociado a otros inmunosupresores. La rapamicina (de Rapanui) es un fármaco descubierto en la Isla de Pascua por el Dr. Shegall en 1973. Se comercializa en Europa en el año 2001. Es una lactona macrocíclica aislada de un hongo (Streptomices Higroscopicus), un agente inmunosupresor, que frena el rechazo mediante la inhibición de la proliferación de los linfocitos T y $\mathrm{B}$.

Su mecanismo de acción se basa en la interferencia de la señal de activación de los linfocitos T mediado por la Interleuquina-2 que se liga a la proteína target of rapamycin (TOR), sin efecto alguno frente a la calcineurina, como el tacrolimus ó la ciclosprina. Esta señal inhibitoria afecta a factores de crecimiento de la célula muscular lisa y fibroblastos.

Se describe como una de las mayores ventajas de la rapamicina, que no es nefrotóxica a las dosis habituales (aunque podría aumentar la toxicidad asociada a la ciclosporina). Entre los efectos adversos se cita la cefalea, poliartralgia, estomatitis, epistaxis, diarrea, acné, etc., destacando entre los más relevantes la hiperlipidemia, la mielodepresión y una inmunodepresión excesiva, si no se ajustan las dosis. Además, respecto a la evolución posquirúrgica, se ha descrito la presencia de edemas y linfoceles, pero se han dado pocas referencias en relación al exudado de las heridas.

Hay que tener en cuenta los factores de riesgo que están implicados en las complicaciones de las heridas quirúrgicas, como la edad, la obesidad, hipoproteinemia, o diabetes, pero para nosotros la rapamicina era un nuevo cambio introducido en la terapia inmunosupresora por lo que decidimos estudiar si el empeoramiento de la herida quirúrgica tenía que ver con su empleo.

Nuestro objetivo es valorar sí, como corresponde a la observación de enfermería, el empeoramiento en la evolución de la herida quirúrgica del postrasplante renal, está en relación con el uso de rapamicina como inmunosupresor.

\section{MATERIAL Y MÉTODO}

Se realizó un estudio epidemiológico observacional de cohortes retrospectivo a un total de 56 pacientes trasplantados en nuestra unidad, desde el año 1999 hasta el 2003, dichos pacientes se distribuyeron en dos grupos:

- Grupo control: Se seleccionan 30 pacientes con la pauta habitual de inmunosupresión: corticoides, ciclosporina o tacrolimus, micofenolato mofetil, etc.

- Grupo rapamicina: 26 pacientes en tratamiento con corticoides, tacrolimus o micofenolato mofetil, pero asociado a rapamicina.

Para el primer grupo se seleccionaron los pacientes de forma aleatoria; en el segundo grupo se incluyó a todos los pacientes a los que se administraba rapamicina. 
Las variables estudiadas son: edad, sexo, diabetes (diabéticos insulino-dependientes antes del trasplante), obesidad (IMC>30), hipoproteinemia (albúmina<3.3 gr/dl), tratamiento antes del trasplante (CAPD o HD).

Respecto a la evolución de la herida se valoró: infección, dehiscencia de sutura y linfocele; se contabilizaron los días respecto a la retirada del redón y la presencia de exudado del orificio post-retirada, de la retirada de las grapas, y de la presencia de exudado de la herida quirúrgica desde el día del trasplante.

El tratamiento de los datos se realizó con el programa estadístico SPSS, tanto para la generación de la base de datos, como para el análisis de los mismos. Para evaluar las diferencias entre los grupos utilizamos la t de Student, chi cuadrado y U de Mann Whitney. Se consideran significativas las diferencias con una $\mathrm{p}<0,05$.

\section{RESULTADOS}

Los factores de edad, sexo, diabetes, IMC, hipoproteinemia y tratamiento pre trasplante con CAPD, se distribuyen en ambos grupos de forma aleatoria. Según se muestra en la tabla 1 son significativas la edad, la diabetes y el IMC. En relación a la herida (tabla 2), la infección (1 caso) y dehiscencia de sutura (3 casos), sólo aparece en el grupo tratado con rapamicina, aunque no es significativo; lo mismo ocurre con el linfocele (4 pacientes en el grupo rapamicina, 2 pacientes en grupo control).

\begin{tabular}{|l|ll|ll|l|}
\hline & Rapamicina $(\mathrm{n}=26)$ & \multicolumn{2}{|c|}{$\begin{array}{c}\text { No Rapamicina } \\
(\mathrm{n}=30)\end{array}$} & Significación \\
\hline Edad & 53,6 & 42,4 & 0,003 \\
\hline Sexo(\% Hombres) & $69,2(18 / 26)$ & 66,7 & $(20 / 30)$ & N.S. \\
\hline Diabéticos & $23,1 \quad(6 / 26)$ & 0 & $(0 / 30)$ & 0,005 \\
\hline IMC $>30$ & $23,1 \quad(6 / 26)$ & 3,3 & $(1 / 30)$ & 0,026 \\
\hline Hipoproteinemia & $23,1(6 / 26)$ & 20,0 & $(6 / 30)$ & N.S. \\
\hline En CAPD pre Tx & $11,5(3 / 26)$ & 20,0 & $(6 / 30)$ & N.S. \\
\hline
\end{tabular}

Tabla 1. Características de los grupos en relación con factores de riesgo.

\begin{tabular}{|l|ll|ll|l|}
\hline & \multicolumn{2}{|l|}{ Rapamicina $(\mathrm{n}=26)$} & \multicolumn{2}{|c|}{$\begin{array}{c}\text { No rapamicina } \\
(\mathrm{n}=30)\end{array}$} & Significación \\
\hline Infección & $3,8 \%$ & $(1 / 26)$ & $0 \%$ & $(0 / 30)$ & N.S. \\
\hline Dehiscencia de sutura & $11,5 \%$ & $(3 / 26)$ & $0 \%$ & $(0 / 30)$ & N.S. \\
\hline Linfocele & $15,4 \%$ & $(4 / 26)$ & $6,7 \%$ & $(2 / 30)$ & N.S. \\
\hline
\end{tabular}

Tabla 2. Variables en relación con la herida quirúrgica.
Tampoco son significativas las diferencias sobre la fecha de la retirada de los redones que coinciden en los dos grupos y sobre el exudado del orificio después de la retirada del redón y de la retirada de las grapas, aunque en pacientes tratados con rapamicina la media es mayor (tabla 3).

\begin{tabular}{|c|l|l|l|l|}
\hline & & Número & \multicolumn{1}{|c|}{ Media días } & Significación \\
\hline Día de retirada del redón & Rapamicina. & 26 & 4,23 & N.S. \\
& No rapamicina & 30 & 3,53 & \\
\hline Exudado del orificio post & Rapamicina. & 26 & 5,81 & N.S. \\
retirada del redón & No rapamicina & 30 & 0,59 & \\
\hline Retirada de grapas & Rapamicina. & 13 & 16,92 & N.S. \\
& No rapamicina & 11 & 16,27 & \\
\hline
\end{tabular}

Tabla 3. Variables numéricas en relación con la herida quirúrgica.

La relación entre los días de exudado de la herida quirúrgica, en el postoperatorio de los pacientes del grupo tratado con rapamicina, es estadísticamente significativa en relación con el grupo que no lo toma, con una $\mathrm{p}=$ 0.016 ( $p<0,05)$ en U de Mann Whitney, como se detalla en la tabla 4 y en la figura 1.

\begin{tabular}{|c|c|l|l|l|}
\hline & & Número & \multicolumn{1}{|c|}{ Media } & Significación \\
\hline $\begin{array}{c}\text { Número dias de } \\
\text { exudado de la herida } \\
\text { quirúrgica }\end{array}$ & Rapamicina. & 26 & 11,96 & $\mathrm{p}=0,016$ \\
No rapamicina & 30 & 2,3 & $\mathrm{p}<0,05$ \\
\hline
\end{tabular}

Tabla 4. Variable numérica respecto al número de días de exudado analizada con la prueba U de Mann - Witney.

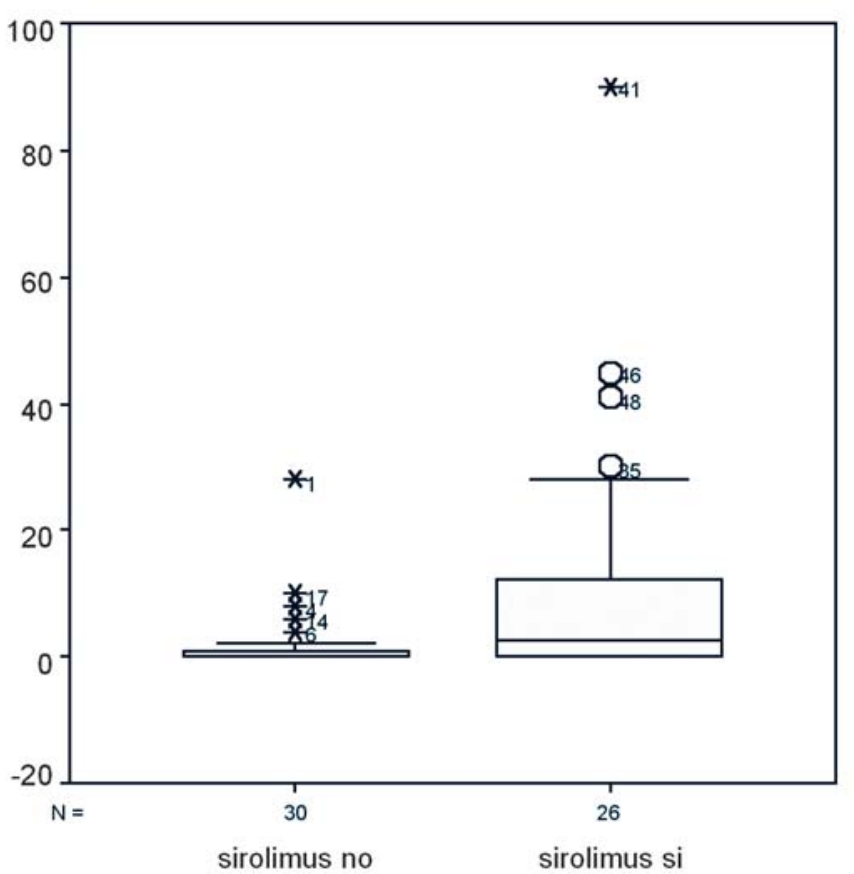

Figura 1. Número de días de exudado de la herida quirúrgica. 


\section{DISCUSIÓN Y CONCLUSIONES}

La rapamicina, por su mecanismo de acción, es un potente agente antiproliferativo que disminuye los fibroblastos e inhibe la angiogénesis, por lo que tiene un efecto negativo en la cicatrización en el post-operatorio. En nuestro grupo ha influido en dicho proceso cicatrizal: la diabetes, la obesidad y la edad. La hipoproteinemia no es valorable, porque todos los pacientes estaban en cifras límite con el rango considerado normal de $3.3 \mathrm{~g} / \mathrm{dl}$. Hay muy pocos casos de infección y dehiscencia de suturas por lo que tampoco parece que sean variables a tener en cuenta. Aunque en pacientes tratados con rapamicina aparece algún caso más de linfocele o exudado del orificio, tampoco estos datos son significativos y lo mismo ocurre con la retirada de las grapas (aunque sólo se ha obtenido datos de 13 y 11 pacientes de cada grupo).

El número de días en que la herida quirúrgica siguió con exudados es mayor en el grupo de pacientes tratados con rapamicina. Nuestros resultados al respecto corresponden a los de otros autores, aunque en ensayos con una muestra mayor este dato tampoco es significativo. Esto puede ser debido a que han considerado como variable, la cicatrización de las heridas a las tres semanas del trasplante. La enfermería detalla en cada turno la situación del paciente y todas sus complicaciones, por eso hemos podido hacer nuestro estudio más minucioso del exudado diario hasta su resolución.

Como actualmente ha aumentado la edad de los pacientes que se trasplantan, tenemos que contar con obesidad y diabetes en muchos de ellos. Si lo asociamos al tratamiento con rapamicina hay que enfrentarse a nuevos retos ya que la rapamicina es un buen inmunosupresor con ventajas en su relación con la menor incidencia tumoral y toxicidad, por lo que se va a seguir usando en el futuro. En este estudio a pesar de los factores que hemos comentado como edad, obesidad y diabetes, se ve una tendencia entre el empeoramiento de la evolución de las heridas quirúrgicas, en el trasplante renal en relación a su cicatrización, debido al uso del tratamiento con rapamicina como inmunosupresor.

Estas reflexiones nos llevan a tomar nuevas medidas en relación con estos pacientes. En las intervenciones quirúrgicas, se puede adoptar la utilización de suturas de reabsorción más lenta, y una cirugía más minuciosa de los vasos linfáticos.
La enfermería, estudiará un protocolo más riguroso de las curas, un seguimiento con medidas específicas de fajado de abdomen, etc., para evitar problemas como la infección, dehiscencia de suturas, etc. El equipo de trasplantes y la enfermería, debe mejorar sus actuaciones en la técnica y los cuidados de los pacientes trasplantados para minimizar las complicaciones.

Se puede completar este estudio en un futuro con más pacientes seleccionados por muestreo estratificado, siendo siempre necesarios unos buenos registros de enfermería que permitan el adecuado análisis de los cuidados que ofrecemos a nuestros pacientes y faciliten la investigación.

\section{BIBLIOGRAFÍA}

1. Botella García J. Trasplante renal. En: Manual de Nefrología Clínica. Masson. Barcelona, 2003; 27: 272279.

2. Crespo M, Campistol JM, Oppenheimer. Protocolos de inmunosupresión postrasplante renal. En: Curso de actualización en trasplante renal, ed. Madrid: Drug Farma; 2002. p. 103-109.

3. Cruzado JM, Grinyo JM. Terapéutica inmunosupresora. En: Nefrología Clínica, Hernando Avendaño L. Madrid: Editorial Médica Panamericana; 2003. p. 896-908.

4. Groth Carl G, Bacckman Lars, Morales JM et al. Sirolimus (Rapamicyn) Based therapy in human renal transplantatión: Similar efficacy and different toxicity compared with cyclosporine. Transplantation 1997; 67(7): 1036-1042.

5. Morales Cerdan JM. Fármacos Inmunosupresores. Protocolos de inmunosupresión y resultados. En: Trasplante Renal. Normas de actuación clínica en nefrología (NAC). Madrid: Harcout S.A. Grafos; 1999. p. 21-39.

6. Stuart M. Flechhner, Ligmei Zhou et al. The impact of sirolimus, micophenolate mofetil, cyclosporine, azathioprine, and steroids on wound healing in 513 kidney-transplant recipients. Transplantation 2003; 76:1729-1734.

7. Valente John F, Hricik Donald et al. Comparasion of Rapamicina rapamicinavs. mycophenolate mofetil on surgical complications and wound healing in adult kidney transplantation. American journal of transplantation 2003; 3:1128-1134. 